\title{
Male accessory breast cancer on the abdominal wall: a case report and literature review
}

This article was published in the following Dove Press journal: OncoTargets and Therapy

\author{
Guo-Bin Zhong' \\ Xin-Qing $\mathrm{Ye}^{2}$ \\ Jian-Lun Liu' \\ Shu-Zhe Xiao ${ }^{3}$ \\ Qing-Hua Huang' \\ Wei Wei' \\ 'Department of Breast Surgery, The \\ Affiliated Tumor Hospital of Guangxi \\ Medical University, Nanning, China; \\ ${ }^{2}$ Department of Pathology, The \\ Affiliated Tumor Hospital of Guangxi \\ Medical University, Nanning, China; \\ ${ }^{3}$ Department of Pediatrics, Guangzhou \\ First Municipal People's Hospital, \\ Guangzhou, China
}

Background: Accessory breast cancer is very rare, particularly in men. Male accessory breast cancer on the abdominal wall has not been documented in the scientific literature so far. We describe a case of male accessory breast cancer on the abdominal wall.

Case presentation: We describe a male patient suffering a swelling and erosive, enlarged, and hardened abdominal wall mass with pain due to abdominal wall accessory breast cancer. The patient had no obvious disease history, and the initial clinical symptom was a small mass on the abdominal wall. B-ultrasound revealed a solid subcutaneous nodule in the right abdomen with a size of $\sim 2.8 \times 2.5 \times 1.5 \mathrm{~cm}$. The abdominal wall tumor resection was performed with local anesthesia. Pathological testing revealed a grade II infiltrating ductal carcinoma derived from the accessory mammary gland (right abdominal wall) with neuroendocrine characteristics, showing ER (100\% strong positive), PR (100\% strong positive), HER-2 (-), ki67 (40\% positive), Syn (+), CgA (+), and GCDFP15 (+).

Conclusion: Nonaxillary accessory breast cancer in males is very rare, with no obvious clinical manifestations, and could be easily ignored. This disease requires great attention from clinicians.

Keywords: accessory breast cancer, accessory mammary gland, breast neoplasms, male, abdominal wall

\section{Background}

Accessory mammary glands are breast tissue in any form other than the normal mammary glands. Accessory mammary glands form when the mammary glands of the embryonic stage (excluding the pair on the chest) are not degraded or are incompletely degraded. Accessory breast cancer is very rare, accounting for $0.3 \%-0.6 \%$ of all breast cancers; it is mostly manifested as an axillary mass and can also grow in any part of the breast line (the line from axilla to groin). ${ }^{1}$ This study described the clinical features and treatment course of a male patient diagnosed with abdominal wall accessory breast cancer, with the goal of contributing to the understanding of this disease.

\section{Case presentation}

A 62-year-old male patient had a mass in his right lower abdominal wall for more than 50 years. He sought treatment due to its progressive growth for 2 years with ulceration and pus for more than 3 months. The patient found a subcutaneous mass on the abdominal wall with no obvious cause more than 50 years ago. The size of the mass was $\sim 1.5 \times 1 \mathrm{~cm}$. The mass could be pushed without tenderness, and the surrounding boundary was unclear. He did not pay attention to the mass. In the past 2 years, the patient felt that the mass had progressively enlarged; its size had increased to $\sim 3 \times 2.5 \mathrm{~cm}$. The texture had gradually hardened and was accompanied by pain.
Correspondence: Wei Wei

Department of Breast Surgery,

The Affiliated Tumor Hospital of Guangxi

Medical University, Nanning, China

Tel +86 I39 28387789

Email13928387789@I63.com 
Three months before seeking treatment, the mass showed swelling and erosion on the surface, with purulent exudates for no obvious incentive.

Physical examination: the patient's two breasts were symmetrical, with no local bulge or erosion and no palpable mass; there were no significantly enlarged lymph nodes palpable in the bilateral axillae and both up and down clavicles. A mass was found in the right lower abdomen with redness, swelling, and erosion on the surface. The mass was $\sim 3 \times$ $2.5 \mathrm{~cm}$ in size. Its texture was hard, the boundary was unclear, and it was not movable with tenderness by pressing. B-ultrasound revealed a subcutaneous nodule in the right abdomen with a size of $\sim 2.8 \times 2.5 \times 1.5 \mathrm{~cm}$.

On February 27, 2017, the patient underwent abdominal mass resection with local anesthesia in a local hospital. Gross examination of the mass showed that a piece of skin tissue had eroded on the skin surface, and the mass was under the incision surface of the skin with a size of $2.8 \times 2.5 \times 1.5 \mathrm{~cm}$; the mass was gray and hard, and the boundary was unclear. Observations by microscopy were as follows: in the subcutaneous tissue, the tumor cells had large nuclei with dark staining; the chromatin was thick, and the nucleoli were obvious; pathological mitosis was shown with less cytoplasm; the tumor cells were arranged in lumps, nests, and glandular tubules in small amounts with necrosis in small amounts (Figure 1). Invasive growth of the tumor tissue was observed, and its boundary was unclear; the infiltration reached the subcutaneous fibrous connective tissue and the superficial skin (Figure 2), invading the vessels and nerves. The pathological diagnosis was grade II infiltrating ductal carcinoma derived from the accessory mammary gland (abdominal wall) with neuroendocrine

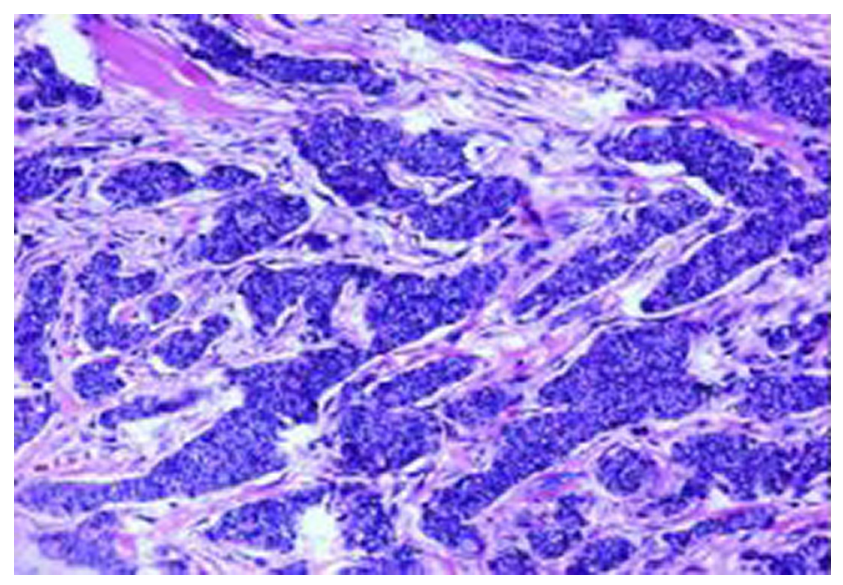

Figure I The tumor cells showing large nuclei with dark staining.

Notes: The chromatin was thick, and the nucleoli were obvious. Pathological mitosis was observed with less cytoplasm. The tumor cells showed infiltrating growth in lumps, nests, and glandular tubules. $\mathrm{H} \& \mathrm{E} \times 100$ magnification.

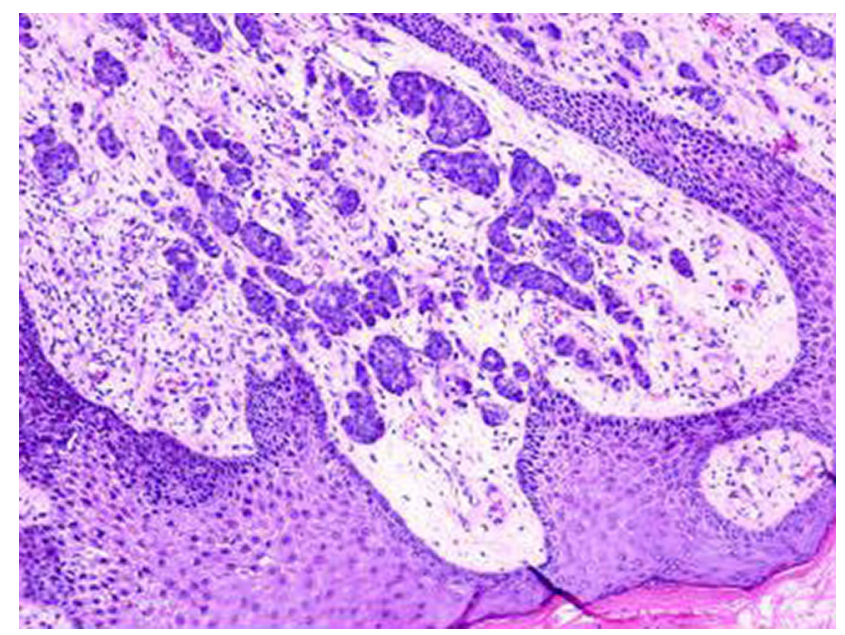

Figure 2 Infiltration of cancer tissue in the dermis of the skin. Note: $\mathrm{H} \& \mathrm{E} \times 100$ magnification.

characteristics, with the immunohistochemistry of ER (100\% strong positive), PR (100\% strong positive), HER-2 (-), ki67 (40\% positive), Syn (+),CgA (+), and GCDFP15 (+) (Figures 3-9).

Postoperative positron emission tomography-computed tomography (CT) showed the following: 1) changes after the tumor resection in the right lower abdominal wall, with no local residual tumor; 2) multiple retroperitoneal and pelvic nodules on both sides, with a maximal size of $2.4 \times 1.8 \mathrm{~cm}$, standardized uptake value $(\mathrm{SUV})_{\max }$ 3.6 and $\mathrm{SUV}_{\text {ave }} 3.2$, which were considered lymph node metastases; 3) multiple nodules in both inguinal regions, with the maximal size of $1.5 \times 1.0 \mathrm{~cm}, \mathrm{SUV}_{\max } 2.7$ and $\mathrm{SUV}_{\text {ave }} 2.4$, which were considered lymph node metastases; 4) no obvious signs of malignant primary tumor in other parts of the body. On March 15, 2017, as shown in

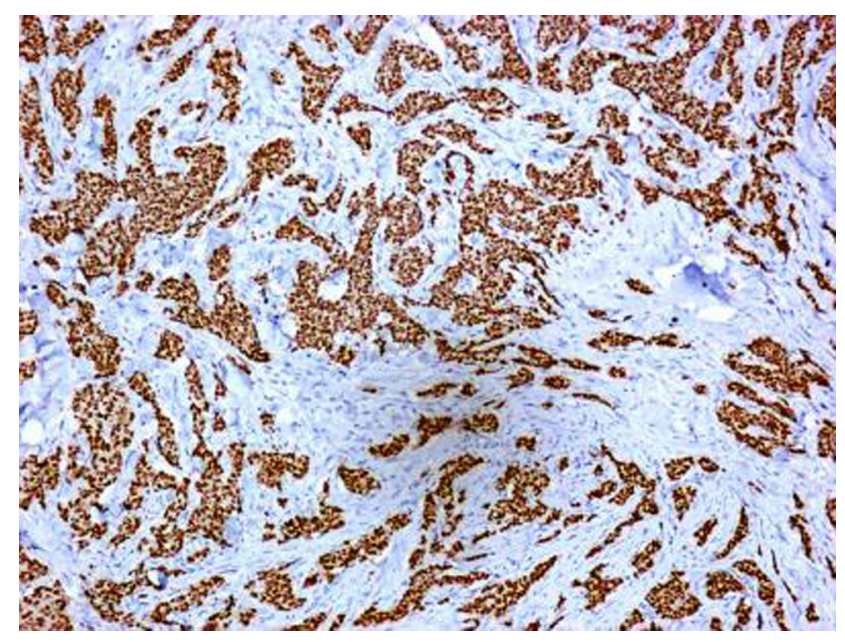

Figure 3 ER of the cancer cell nuclei ( $100 \%$ strong positive); envision method $\times 100$ magnification. 


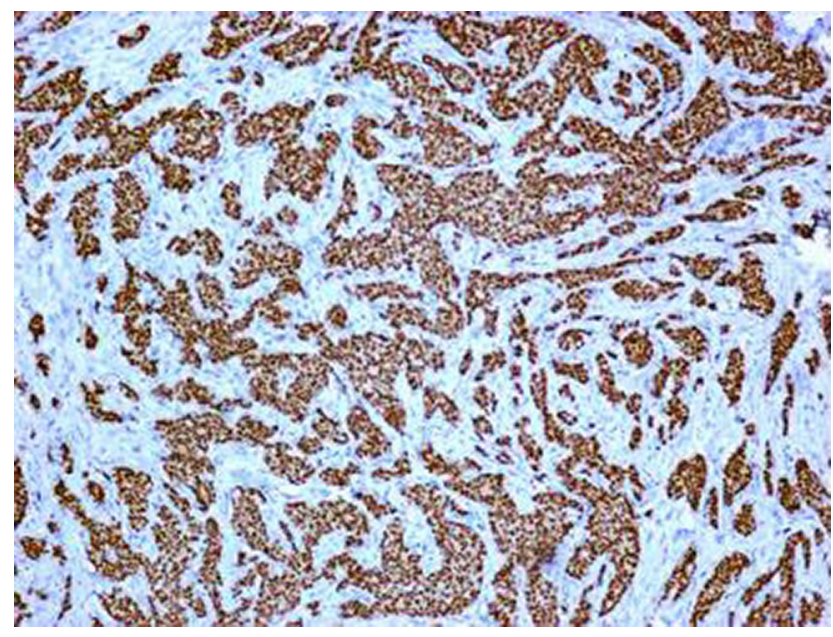

Figure 4 PR of the cancer cell nuclei ( $100 \%$ strong positive); envision method $\times 100$ magnification.

Figure 10, CT showed postoperative changes on the right lower abdominal wall; the structure of the surgical area was disordered, and the skin was slightly thicker, with a subcutaneous patchy high-density shadow; a few lymph nodes were observed near the bilateral groin and iliac vessels; and some patchy low lesions were uneven in their internal density, including a large lesion $\sim 1.7 \times 2.3 \mathrm{~cm}$ in size. A definitive diagnosis of accessory breast cancer at the right abdominal wall with bilateral inguinal and peritoneal lymph node metastases in stage VI was obtained. On March 28, 2017, radiotherapy was performed with the following dosing: abdominal surgical area of the original tumor: $6 \mathrm{MeV}$ electron beam, $50 \mathrm{~Gy} / 25$ f; pelvic area: $6 \mathrm{Mev}-\mathrm{X}$ ray, PGTV: 50 Gy/25 f, PCTV: 45 Gy/25 f. On May 18, 2017, as shown in Figure 11, the CT reexamination revealed shrinkage of several enlarged lymph nodes around the bilateral groin and

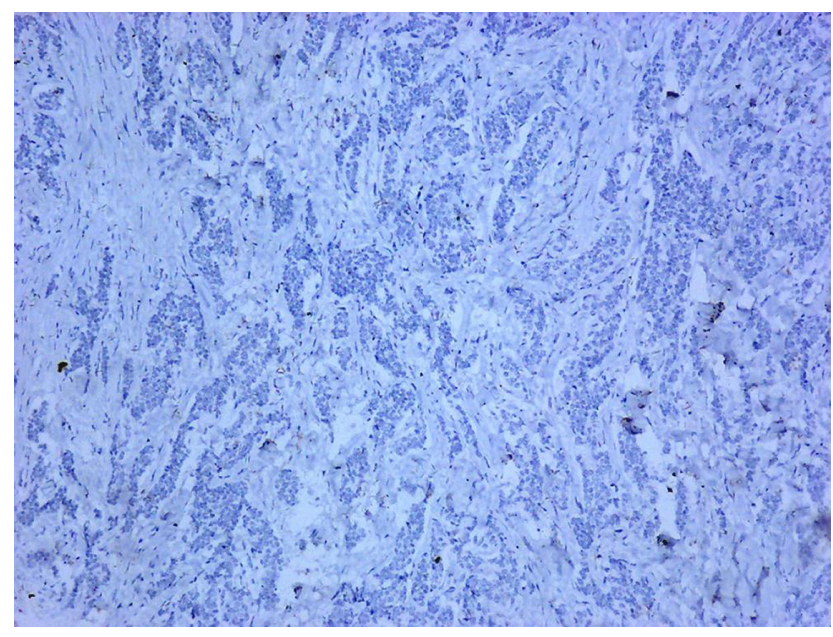

Figure 5 HER-2 negative; envision method $\times 100$ magnification.

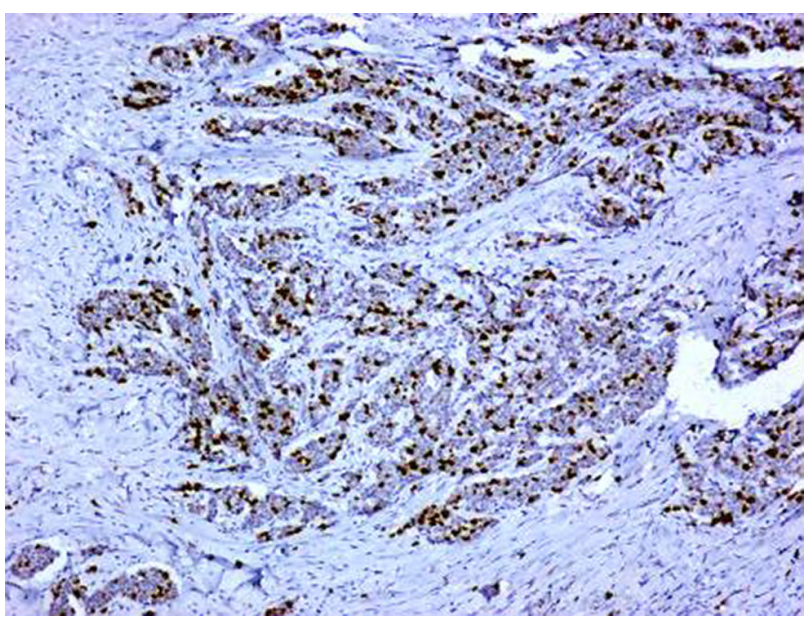

Figure $6 \mathrm{Ki} 67$ of the cancer cell nuclei (40\%, strong positive); envision method $\times 100$ magnification.

iliac vessels and some patchy low lesions with uneven internal density, including a large lesion $\sim 2.0 \times 1.4 \mathrm{~cm}$ in size. After radiotherapy, the patient was treated with tamoxifen $10 \mathrm{mg}$ bid until now. The CT reexamination on August 1, 2017, as shown in Figure 12, showed that multiple lymph nodes near the bilateral groin and iliac vessels were smaller than before, and some of them had low-density necrosis, with the largest one $\sim 1.7 \times 1.2 \mathrm{~cm}$ in size. As of this writing, the patient is in stable condition.

\section{Discussion}

Male breast cancer is rare in clinical practice, with a low incidence, accounting for $<1 \%$ of all breast cancers and $<1 \%$ of all male malignancies. ${ }^{2}$ However, these incidences have shown an increasing trend in recent years, ${ }^{3}$ and the incidence of male breast cancer has increased by $26 \%$ over 25 years. ${ }^{4,5}$

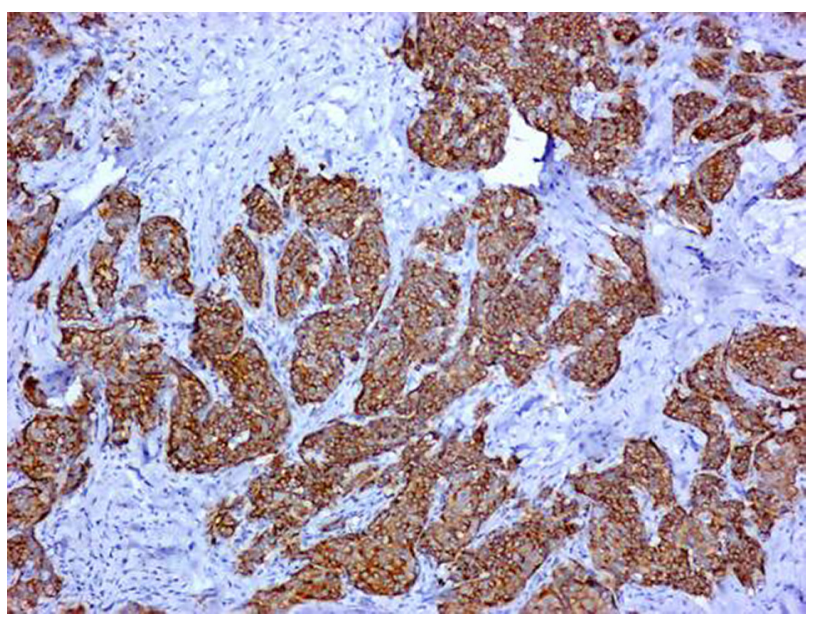

Figure 7 Syn of the cancer cell cytoplasm (diffuse positive); envision method $\times 200$ magnification. 


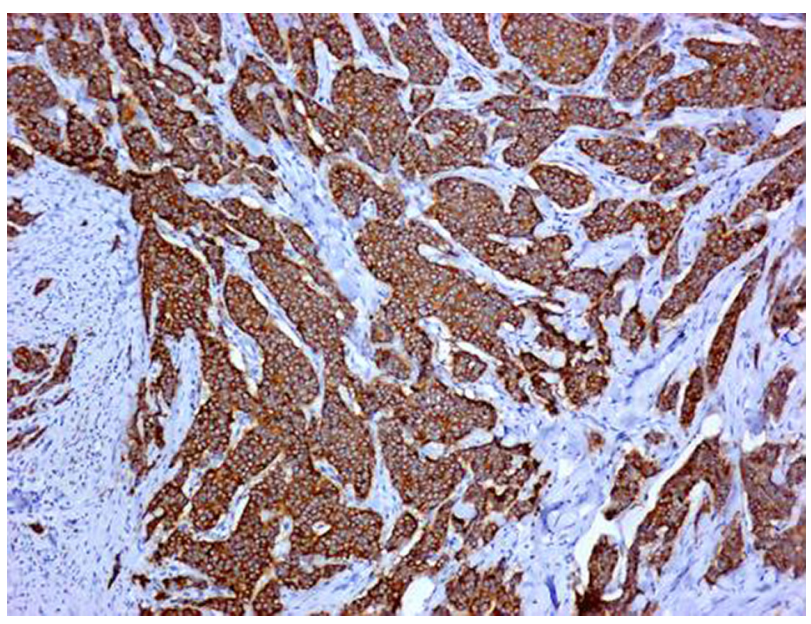

Figure $8 \mathrm{CgA}$ of the cancer cell cytoplasm (diffuse positive); envision method $\times 200$ magnification.

It has been reported in the literature that the incidence of male accessory breast cancer is higher than that of breast cancer. ${ }^{6,7}$ A case of male accessory breast cancer in the left arm was reported previously, ${ }^{8}$ but male accessory breast cancer on the abdominal wall has not been documented.

The precise cause of male accessory breast cancer is not yet fully understood, but any risk factor that may induce breast cancer can trigger accessory breast cancer. ${ }^{9}$ The main risk factors include the following. 1) Genetic factors: BRCA1, BRCA2, P53, PTEN, PALB2, RAD50, and CDH1 are the susceptibility genes for breast cancer development, especially BRCA2, which plays an important role in the susceptibility of male breast cancer; ${ }^{10} 2$ ) Hormonal factors: the increase in estrogen's effect or an imbalance in estrogen and androgen levels is a risk factor for male breast cancer; ${ }^{11}$ 3) Environmental factors: electromagnetic field exposure

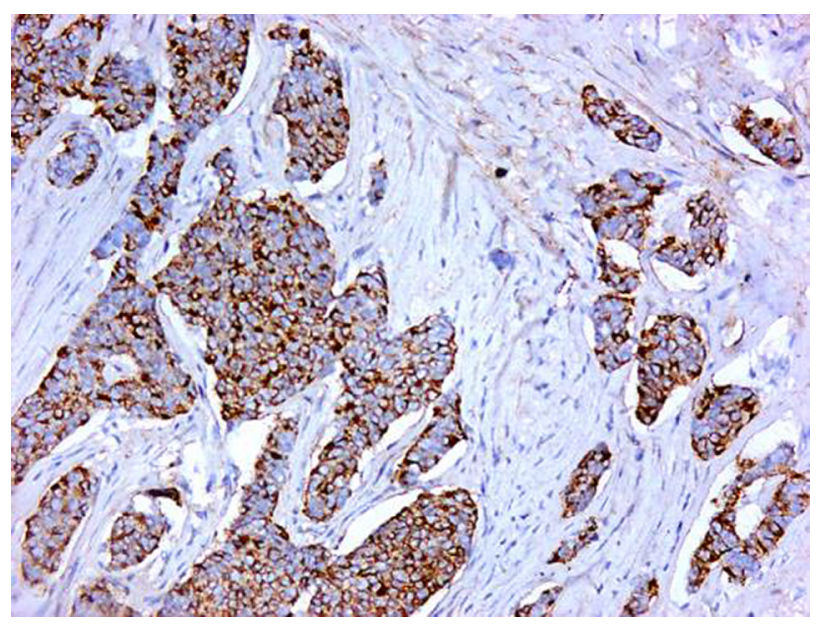

Figure 9 GCDFPI5 of the cancer cell cytoplasm (diffuse positive); envision method $\times 200$ magnification.

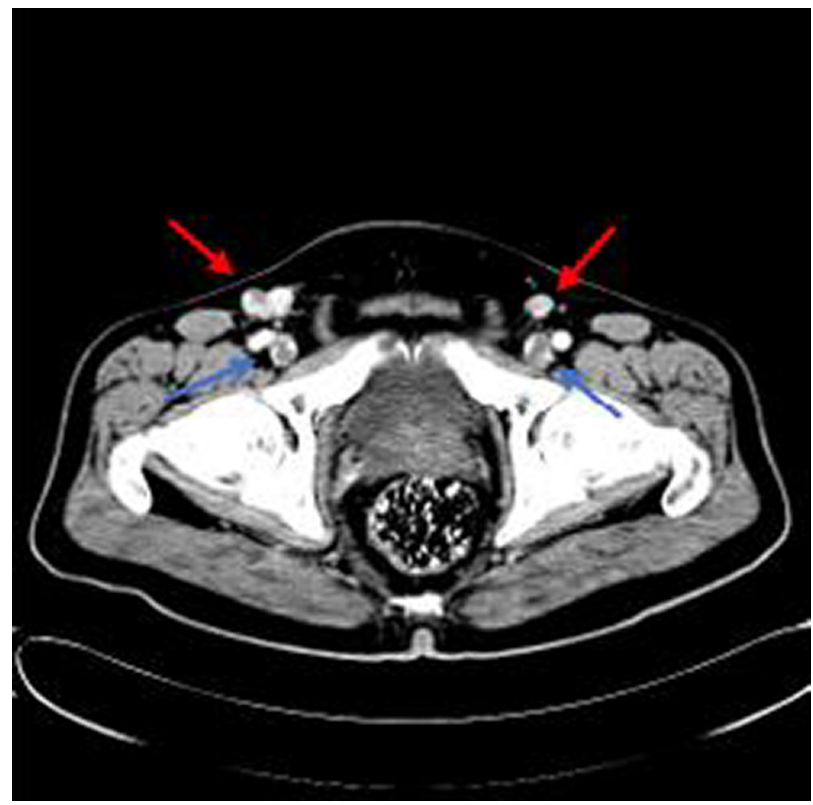

Figure 10 CT on March 15, 2017, shows the enlarged inguinal lymph nodes (red arrows) and enlarged lymph nodes around the iliac vessels (blue arrows). Abbreviation: CT, computed tomography.

may be associated with the increased risk of male breast cancer despite the arguments raised..$^{12}$ In addition, the long-term exposure to high temperature or environments with styrene and formaldehyde can also induce male breast cancer; and $^{13}$ 4) Other factors such as alcoholism, obesity, depression, drugs, and lack of exercise are also contributors.

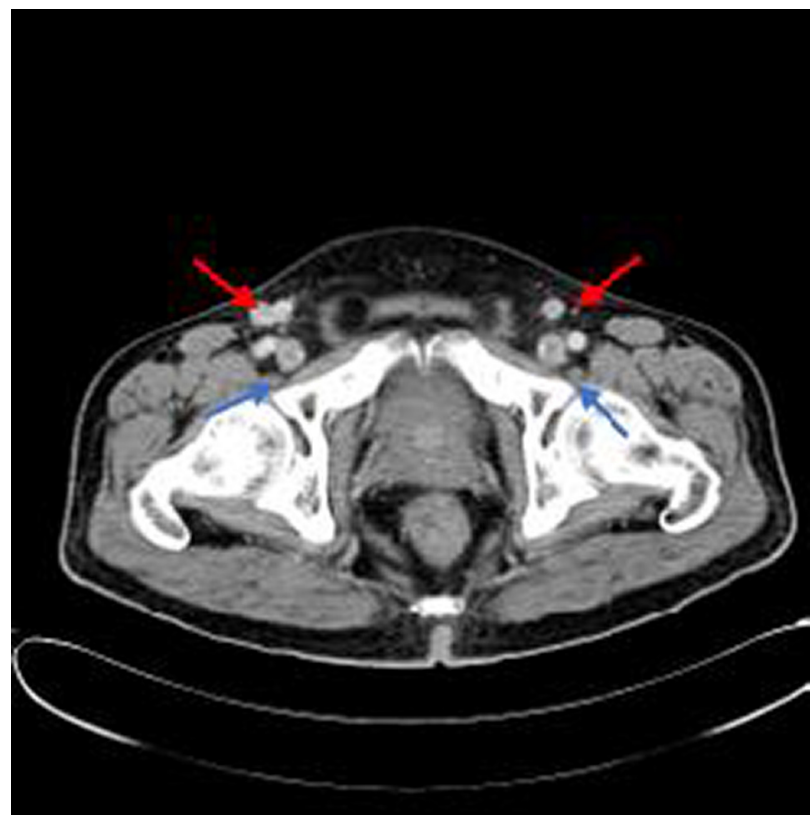

Figure II CT on May 18, 2017, shows the enlarged inguinal lymph nodes (red arrows) and enlarged lymph nodes around the iliac vessels (blue arrows). Abbreviation: CT, computed tomography. 


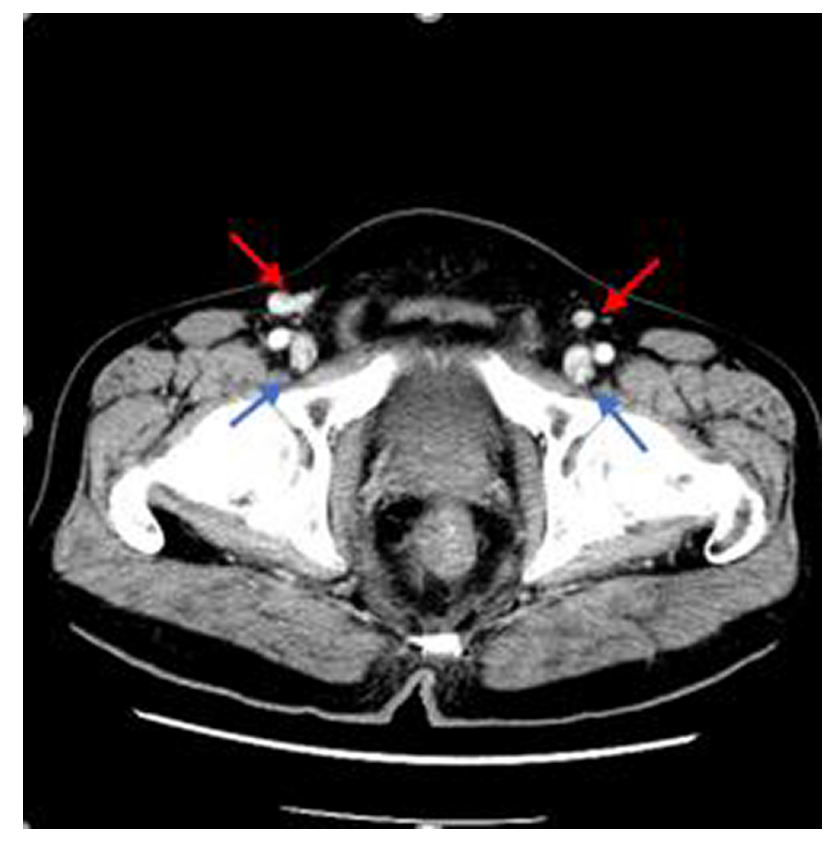

Figure I2 CT on August I, 2017, shows the enlarged inguinal lymph nodes (red arrows) and enlarged lymph nodes around the iliac vessels (blue arrows). Abbreviation: CT, computed tomography.

For the diagnosis of accessory breast cancer, the clinician should first have enough awareness and understanding of accessory mammary glands and accessory breast cancer. Since accessory mammary glands are located in the subcutaneous and dermal tissues of the skin, ${ }^{14}$ accessory breast cancers are often located in superficial areas, have hard textures and unclear boundaries, and can protrude and adhere to the skin and even cause ulceration. For masses appearing on the breast line (the line from axilla to groin), accessory mammary glands should be considered. Second, modern auxiliary examination methods should be used. In related auxiliary examinations, ultrasound examination may be preferred. The ultrasonography of accessory breast cancer is typically characterized by a hypoechoic mass with uneven echogenicity and a crab foot-shaped edge. ${ }^{15}$ Madej et al ${ }^{16}$ believed that accessory mammary glands do not develop on molybdenum target imaging. ${ }^{6}$ Currently, the value of CT scan is not yet clear, but it is conducive to differential diagnosis and staging assessment. It has been reported that MRI can increase the detection rate of accessory breast cancer. ${ }^{17}$ Imaging of accessory mammary glands on MRI is characterized by low signal on a T1-weighted image and high signal on a T2-weighted image or enhanced kinetics consistent with the enhancement of the tumor with gadolinium contrast agent. The gold standard for the diagnosis of accessory breast cancer is still pathological examination. Preoperative thick needle biopsy is conducive to confirm the diagnosis and to guide the development of a treatment plan. ${ }^{18}$ In addition to the typical histological characteristics of breast cancer, the positive expression of breast-derived immunohistochemical markers can also further confirm the diagnosis.

The definitive diagnosis of male accessory breast cancer on the abdominal wall in this reported case had several diagnostic keys: the tumor was located on the breast line and was not associated with the normal mammary glands; the pathological diagnosis showed a grade II infiltrating ductal carcinoma derived from the accessory mammary glands (abdominal wall) with neuroendocrine characteristics; and nonbreast cancer metastasis was confirmed. At the same time, rare tumors on the abdominal wall, such as abdominal wall metastasis and abdominal hernia, should be carefully distinguished.

The treatment of accessory breast cancer is similar to that of breast cancer and follows the principle of comprehensive treatment, with surgery as the main treatment, supplemented with chemotherapy, radiotherapy, endocrine therapy, and molecular targeted therapy. According to Madej et al, ${ }^{16}$ the successful experience of breast conservative surgery for breast cancer can also be applied to surgery for accessory breast cancer to reduce the range of resection. If the diagnosis of accessory breast cancer in the axillary area is clear, an extended resection of the accessory mammary gland region with axillary lymph node dissection should be performed. ${ }^{19}$ It is not necessary to remove the ipsilateral breast unless the accessory breast cancer is very close to the mammary gland or is connected to the mammary gland or unless invasion of cancer cells in the ipsilateral mammary gland has been observed. If the preoperative tumor is large, neoadjuvant chemotherapy or endocrine therapy can be performed first, and the surgery can be performed after the tumor is significantly reduced. For patients with accessory breast cancer, postoperative conventional radiotherapy is recommended to reduce the risk of local recurrence. ${ }^{7}$ After chemotherapy and endocrine therapy, the indications for targeted therapy are the same as those for breast cancer. The patient in this case report received tumor resection in a local hospital, but the margins were not sufficient. Postoperative examination revealed metastases in bilateral inguinal and pelvic and intraabdominal lymph nodes. This patient was in an advanced stage and should be treated with systemic therapy. Because the hormone receptors were highly expressed, endocrine therapy was provided.

Due to the lack of understanding of accessory breast cancer among clinicians, definitive diagnoses for most accessory breast cancers are delayed, misdiagnosed, and missed. 
In particular, accessory breast cancer in males has a high misdiagnosis rate. ${ }^{20}$ In patients with local infiltration, lymph node metastasis, or even distant metastases, the prognosis is poor. ${ }^{7}$ However, patients with accessory breast cancer undergoing surgery-based standardized treatment showed a relatively high survival rate in the retrospective studies. The 5 -year survival rates of the patients with accessory breast cancer were $41.7 \%$ in the Cancer Hospital of the Chinese Academy of Medical Sciences and 35.3\% in Tianjin Cancer Hospital. ${ }^{14}$ Researchers also found that the prognosis of accessory breast cancer was not worse than that of breast cancer in the same time period. ${ }^{6,19}$

\section{Conclusion}

The definitive diagnosis of accessory breast cancer in this case suggested that physicians and patients should pay attention to the development of any unexplained lumps in any part of the body. Moreover, it is necessary to strengthen studies related to accessory breast diseases. Modern medical examination methods should be used to achieve a clear diagnosis in the early stage and to develop an individualized treatment plan in the early stage, thus improving the survival rate of patients with accessory breast cancer. It has been reported that the incidence of accessory breast cancer is significantly higher than that of accessory benign tumors, ${ }^{21,22}$ therefore, we recommend that individuals with small masses in accessory mammary glands and no symptoms should pay adequate attention, with regular checkups. Moreover, accessory mammary glands with large tumors or related symptoms should be resected as early as possible. In addition, we advocate the active promotion of male breast cancer knowledge for early detection, early diagnosis, and early treatment.

\section{Abbreviations}

CT, computed tomography; ER, estrogen receptor; HER-2, human epidermal growth factor receptor-2; PR, progesterone receptor; PET, positron emission tomography; SUV, standardized uptake value.

\section{Acknowledgment}

This study was supported by grant (no Z2016493) from the Guangxi Zhuang Autonomous Region Health and Family Planning Commission. Xin-Qing Ye is the co-first author.

\section{Ethics approval and consent to participate}

The study was approved by the ethical standards of the Affiliated Tumor Hospital of Guangxi Medical University.
Written informed consent was obtained from the patient for study entry.

\section{Author contributions}

All authors contributed toward data analysis, drafting and critically revising the paper and agree to be accountable for all aspects of the work.

\section{Disclosure}

The authors report no conflicts of interest in this work.

\section{References}

1. Kitamura K, Kuwano H, Kiyomatsu K, et al. Mastopathy of the accessory breast in the bilateral axillary regions occurring concurrently with advanced breast cancer. Breast Cancer Res Treat. 1995;35(2): 221-224.

2. Siegel R, Naishadham D, Jemal A. Cancer statistics for Hispanics/ Latinos, 2012. CA Cancer J Clin. 2012;62(5):283-298.

3. Doyle S, Steel J, Porter G. Imaging male breast cancer. Clin Radiol. 2011;66(11):1079-1085.

4. Ottini L. Male breast cancer: a rare disease that might uncover underlying pathways of breast cancer. Nat Rev Cancer. 2014;14(10):643-644.

5. Giordano SH, Cohen DS, Buzdar AU, Perkins G, Hortobagyi GN. Breast carcinoma in men: a population-based study. Cancer. 2004;101(1): $51-57$.

6. Nihon-Yanagi Y, Ueda T, Kameda N, Okazumi S. A case of ectopic breast cancer with a literature review. Surg Oncol. 2011;20(1):35-42.

7. Yamamura J, Masuda N, Kodama Y, et al. Male breast cancer originating in an accessory mammary gland in the axilla: a case report. Case Rep Med 2012. 2012;286210.

8. Yang HS, Liu XW, Yang JS. Male left arm accessory breast cancer: a report of one cases. Jilin Med. 2000;144.

9. Zhang S, Wang MS, Yh Y. Diagnosis and treatment of accessory breast cancer. Chinese J Cancer Prev Treat. 2014;21: $1208-1212$.

10. Rizzolo P, Silvestri V, Tommasi S, et al. Male breast cancer: genetics, epigenetics, and ethical aspects. Ann Oncol. 2013;24(Suppl 8): viii75-viii82.

11. Krause W. Male breast cancer - an andrological disease: risk factors and diagnosis. Andrologia. 2004;36(6):346-354.

12. Sun JW, Li XR, Gao HY, et al. Electromagnetic field exposure and male breast cancer risk: a meta-analysis of 18 studies. Asian Pac J Cancer Prev. 2013;14(1):523-528.

13. Coyle YM, Hynan LS, Euhus DM, Minhajuddin AT. An ecological study of the association of environmental chemicals on breast cancer incidence in Texas. Breast Cancer Res Treat. 2005;92(2):107-114.

14. Chang H, Tian XS, Zhang LP. Axillary accessory breast cancer: report of four cases. Chinese J of Oncol. 2004;31:559.

15. Wang HT, Duan JJ. Clinical analysis, diagnosis and treatment of 22 cases of accessory breast cancer. Nat Med J China. 2015;95:260-263.

16. Madej B, Balak B, Winkler I, Burdan F. Cancer of the accessory breast - a case report. Adv Med Sci. 2009;54(2):308-310.

17. Capobianco G, Spaliviero B, Dessole S, et al. Lymph node axillary metastasis from occult contralateral infiltrating lobular carcinoma arising in accessory breast: MRI diagnosis. Breast J. 2007;13(3):305-307.

18. Akbulut M, Zekioglu O, Kapkac M, Ozdemir N. Fine needle aspiration cytologic features of medullary carcinoma of the breast: a study of 20 cases with histologic correlation. Acta Cytol. 2009;53(2): $165-173$.

19. Youn HJ, Jung SH. Accessory breast carcinoma. Breast Care. 2009; 4(2):104-106. 
20. Visconti G, Eltahir Y, van Ginkel RJ, et al. Approach and management of primary ectopic breast carcinoma in the axilla: where are we? A comprehensive historical literature review. J Plastic Reconstruct Aesthetic Surg. 2011;64(1):e1-e11.

21. Kogut M, Bidier M, Enk A, Hassel JC. Axillary accessory breast tissue - case report and review of literature. J Dtsch Dermatol Ges. 2014;12(6):499-500.
22. Zhang SP, Xun XQ, Huang T. Retrospective review of the clinical data of secondary breast cancer. Cancer Res Prev Treat. 2013;40:391-394.

\section{Publish your work in this journal}

OncoTargets and Therapy is an international, peer-reviewed, open access journal focusing on the pathological basis of all cancers, potential targets for therapy and treatment protocols employed to improve the management of cancer patients. The journal also focuses on the impact of management programs and new therapeutic agents and protocols on

\section{Dovepress}

patient perspectives such as quality of life, adherence and satisfaction. The manuscript management system is completely online and includes a very quick and fair peer-review system, which is all easy to use. Visit http://www.dovepress.com/testimonials.php to read real quotes from published authors.

Submit your manuscript here: http://www.dovepress.com/oncotargets-and-therapy-journal 